\title{
Levantamento de seio maxilar seguido de instalação imediata de implante do tipo cone morse: relato de caso
}

Maxillary sinus survey followed with immediate cone morse implant installation: case report Elevación seno maxilar seguida con instalación inmediata del implante cone morse: reporte de caso Camila Melo PEREIRA ${ }^{1}$

Layla Louise de Amorim ROCHA ${ }^{1}$

Matheus Francisco Barros RODRIGUES ${ }^{1}$

Cristofe Coelho Lopes da ROCHA ${ }^{2}$

Rimsky Coelho Lopes da ROCHA ${ }^{3}$

${ }^{1}$ Curso de Odontologia, Faculdade Cathedral,69307-053 Boa Vista - RR, Brasil

${ }^{2}$ Departamento infraestrutura, Instituto Federal, 69303-340 Boa Vista - RR,Brasil

${ }^{3}$ Centro de Pesquisas Odontológicas, São Leopoldo Mandic, 69305-455 Boa Vista RR, Brasil

\section{Resumo}

A cirurgia de levantamento de seio maxilar visa a corrigir a insuficiência óssea na região posterior da maxila e viabilizar a instalação dos implantes em casos de perda dentária que geram grande reabsorção óssea e pneumatização do seio maxilar. O presente estudo tem por objetivo relatar um caso de levantamento de seio maxilar seguido de instalação imediata de implante do tipo cone-morse, demonstrando a eficiência da técnica cirúrgica através da janela lateral e a eficiência do implante em sua instalação concomitante a elevação do seio. O levantamento de seio maxilar é realizado com frequência, porém há contraindicações desse procedimento para pacientes com patologia sinusal, fumantes excessivos, portadores de problemas psicológicos que impossibilitam tratamento em longo prazo, entre outros. Pôde-se concluir que o levantamento de seio maxilar com a instalação imediata de implante cônico é eficaz. Para alcançar bons resultados é necessário levar em consideração alguns fatores que são essenciais para o sucesso destes procedimentos, são eles: a quantidade de remanescente ósseo, a estabilidade primária do implante no momento da instalação, a manutenção da integridade da membrana sinusal, a saúde do seio maxilar prévio e o tipo de conexão do implante.

Descritores: Levantamento do Assoalho do Seio Maxilar; Seio Maxilar; Procedimentos Cirúrgicos Bucais.

\section{Abstract}

Maxillary sinus lift surgery aims to correct bone insufficiency in the posterior region of the maxilla and enable the installation of implants in cases of tooth loss that generate major bone resorption and pneumatization of the maxillary sinus. The present study aims to report a case of maxillary sinus lift followed by immediate installation of a cone-morse type implant, demonstrating the efficiency of the surgical technique through the side window and the efficiency of the implant in its installation concomitant to elevation of the sinus. The lifting of the maxillary sinus is performed frequently, but there are contraindications for this procedure for patients with sinus pathology, excessive smokers, people with psychological problems that make long-term treatment impossible, among others. It was concluded that the maxillary sinus lift with the immediate installation of a conical implant is effective. To achieve good results, it is necessary to take into account some factors that are essential for the success of these procedures, they are: the amount of bone remaining, the primary stability of the implant at the time of installation, the maintenance of the integrity of the sinus membrane, the health of the anterior maxillary sinus and the type of implant connection.

Descriptors: Sinus Floor Augmentation; Maxillary Sinus; Oral Surgical Procedures.

\section{Resumen}

La cirugía de elevación del seno maxilar tiene como objetivo corregir la insuficiencia ósea en la región posterior del maxilar y posibilitar la instalación de implantes en casos de pérdida dentaria que generen mayor reabsorción ósea y neumatización del seno maxilar. El presente estudio tiene como objetivo reportar un caso de elevación del seno maxilar seguido de la instalación inmediata de un implante tipo cono-morse, demostrando la eficiencia de la técnica quirúrgica a través de la ventana lateral y la eficiencia del implante en su instalación concomitante a la elevación del seno. El lifting del seno maxilar se realiza con frecuencia, pero existen contraindicaciones para este procedimiento para pacientes con patología sinusal, fumadores excesivos, personas con problemas psicológicos que imposibilitan el tratamiento a largo plazo, entre otros. Se concluyó que la elevación del seno maxilar con la instalación inmediata de un implante cónico es efectiva. Para lograr buenos resultados, es necesario tener en cuenta algunos factores que son fundamentales para el éxito de estos procedimientos, son: la cantidad de hueso remanente, la estabilidad primaria del implante al momento de la instalación, el mantenimiento de la integridad de la membrana sinusal, la salud del seno maxilar anterior y el tipo de conexión del implante.

Descriptores: Elevación del Piso del Seno Maxilar; Seno Maxilar; Procedimientos Quirúrgicos Orales.

INTRODUÇÃO

A estética facial, em especial a aparência de lábios e dentes, interferem diretamente na vida social das pessoas ${ }^{1}$. A ausência de um elemento dentário leva os pacientes a buscarem alternativas imediatas para a reposição do órgão perdido2. Quando ocorre a perda de um ou mais dentes, inicia-se um processo de remodelação óssea que regularmente impossibilita a reabilitação dos espaços edêntulos com implantes ${ }^{2}$. Desse modo devem-se utilizar meios de reposição óssea com materiais autógenos, heterógenos ou aloplásticos ${ }^{3}$.

A perda de dentes na maxila posterior além de gerar reabsorção óssea natural, pode ocasionar a pneumatização do seio maxilar $^{4-6}$. Ademais, o osso da região posterior da maxila possui baixa densidade óssea, com osso trabeculado poroso e cortical óssea delgada, constituindo fatores que inviabilizam a instalação dos implantes ${ }^{7,8}$.

A cirurgia de levantamento de seio maxilar visa a corrigir a insuficiência óssea da maxila e viabilizar a instalação dos implantes ${ }^{3,4,7}$. A técnica consiste em aumentar a altura óssea vertical ao reposicionar o assoalho do seio, elevando cuidadosamente a membrana sinusal, criando assim um espaço apropriado 
para acomodar o enxerto ósseo e o implante ${ }^{3,9}$.

Apesar de 0 enxerto autógeno ser considerado o padrão ouro para reconstrução do osso por suas propriedades osteocondutoras e osteogênicas, seu uso requer maior tempo cirúrgico e a quantidade de material disponível é limitada ${ }^{3}$. Frente aos fatores supracitados, diversos biomateriais foram desenvolvidos e dentre eles o xenoenxerto que é proveniente principalmente do osso bovino, possui arquitetura óssea semelhante à humana, é seguro, biocompatível, não proporciona morbidades ao paciente e apresenta resultados satisfatórios a longo prazo ${ }^{3}$.

A elevação do seio maxilar pode ser realizada através de duas técnicas, são elas: a técnica da janela lateral e a técnica minimamente traumática por abordagem via alveolar ${ }^{9}$. O acesso por meio da técnica lateral pode ser realizado com broca rotatória cirúrgica, piezoelétrico ou trephines cirúrgicos ${ }^{9}$. A eleição da técnica a ser utilizada dependerá da altura óssea residual remanescente ${ }^{9}$.

O presente estudo tem por objetivo relatar um caso de levantamento de seio maxilar seguido de instalação imediata de implante do tipo cone morse, demonstrando a eficiência da técnica cirúrgica através da janela lateral e a eficiência do implante em sua instalação concomitante a elevação do seio. O estudo tem como justificativa contribuir com a tomada de decisão do cirurgião-dentista na escolha da melhor técnica, levando em consideração o tipo de enxerto empregado e o tipo de implante a ser utilizado.

\section{CASO CLÍNICO}

Paciente do gênero feminino, 39 anos, compareceu à clínica odontológica privada relatando queixa estética e dificuldade de mastigação na região dos dentes 25 e 26 . Na anamnese, paciente relatou não apresentar nenhum problema de saúde geral e não fazer uso de qualquer medicamento, sendo classificada como ASA I. Após avaliação clínica e radiográfica (tomografia computadorizada), constatou-se pneumatização do seio maxilar nesta região (Figura 1). A tomografia computadorizada revelou um seio maxilar saudável e sem presença de qualquer alteração. O remanescente ósseo na região do dente 25 apresentava $6,80 \mathrm{~mm}$ de altura residual e na região do elemento 26 apenas $1 \mathrm{~mm}$ de altura óssea (Figura 1). Nessas condições a estabilidade primária com a instalação imediata do implante só seria permitida na região do dente 25 e a instalação do implante na região do elemento 26 somente após a completa cicatrização da cirurgia de levantamento de seio maxilar. Após anestesia infiltrativa da região com articaína 4\% e epinefrina 1.100.000 (DFL, Brasil), procedeu-se a incisão supracrestal do dente 24 a 27 , intrasulcular na face vestibular do dente 24 e incisão oblíqua na mesial do dente 24 , com lâmina de bisturi 15C (Embramac, Campinas, São Paulo, Brasil). Após a confirmação da incisão com dissector, um retalho único foi rebatido. A janela da osteotomia foi realizada cuidadosamente através da técnica lateral na parede anterior vestibular do seio (Figura 2) com broca esférica diamantada $n^{\circ} 08$, a $7 \mathrm{~mm}$ do início do rebordo ( $1 \mathrm{~mm}$ do assoalho de seio maxilar), peça reta 1:1 (NSK), em 20.000 rpm e irrigação a $80 \%$.

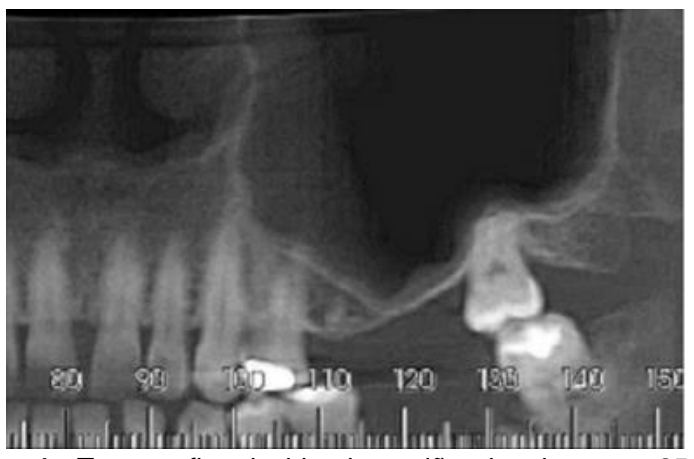

Figura 1: Tomografia elucidando região do elemento $25 \mathrm{com}$ altura óssea residual relativamente maior que na região do 26 e pneumatização do seio maxilar.

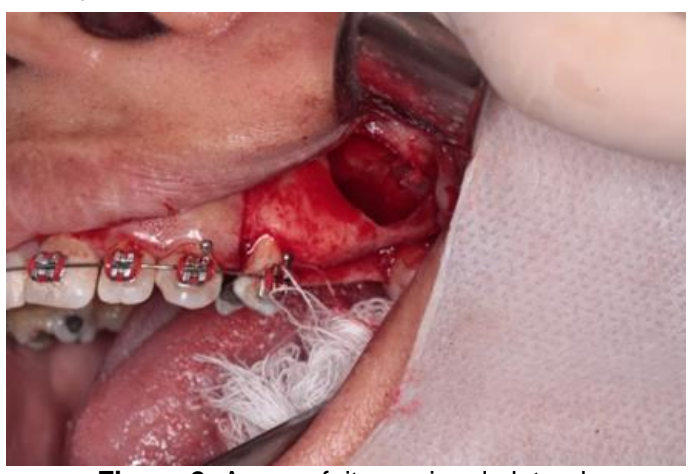

Figura 2: Acesso feito por janela lateral.

Após a visualização da membrana em toda a extensão da janela cirúrgica, foi pressionado o cabo do espelho contra a parte óssea central da janela para constatar a conclusão da osteotomia. Iniciou-se então, o descolamento da membrana com curetas específicas sem corte. Concluído o descolamento da membrana, posicionou-se uma cureta para manter a membrana elevada, evitando qualquer dano durante os procedimentos de perfuração óssea para instalação do implante. Não houve perfuração da membrana sinusal durante o procedimento. Foi selecionado um implante com características indicadas para osso tipo III e IV (Titaoss max cma, cone Morse, Intraoss) de 4.3 de diâmetro por $8 \mathrm{~mm}$ de comprimento. Foi utilizado o kit cirúrgico do mesmo fabricante. A 
rotação de perfuração foi de 800rpm. O biomaterial de escolha foi o Bonefill Mix® (Bionnovation, Brasil) (2 vidros), umidecido em solução salina. Após a conclusão da perfuração para a instalação do implante, a porção mesial, distal e palatina do assoalho de seio maxilar foi preenchida com o biomaterial, acomodado na cavidade sem compactação (Figura 3). O implante foi instalado com rotação de 30rpm, 2 $\mathrm{mm}$ infra ósseo. O torque final foi de $30 \mathrm{~N} . \mathrm{cm}$, aferido com catraca torquímetro.

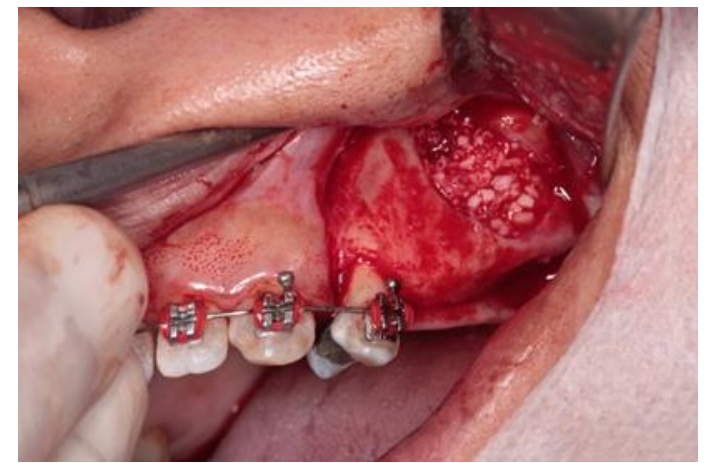

Figura 3: Janela óssea preenchida com enxerto bovino.

O restante da cavidade foi preenchido com biomaterial sem excessos por vestibular. $\mathrm{O}$ parafuso de cobertura foi instalado com chave digital 1.2. O tecido foi reposicionado sem tensão. A sutura foi feita com fio Mononylon 4.0, colchoeiro vertical na distal do dente 25 e mesial do 26 , e os pontos simples nas demais regiões. Foi realizada radiografia periapical pósoperatória imediata (Figura 4).

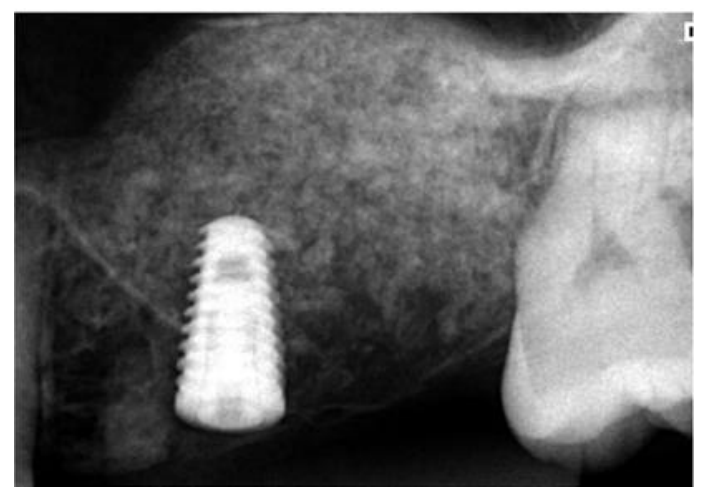

Figura 4: Implante instalado na região do dente 25 apresentando área adjacente preenchida com enxerto ósseo.

A paciente recebeu instruções de higiene oral e cuidados pós-operatórios. A medicação pós-operatória incluiu Amoxicilina $500 \mathrm{mg}$ de 08/08 horas, durante 14 dias; Nimesulid 100mg de 12/12 horas, durante 4 dias; e bochechos suaves com solução de Digluconato de Clorexidine $0,12 \%, 3$ vezes ao dia (somente 48 horas após a cirurgia). A sutura foi removida 15 dias após a cirurgia. No período pós-operatório não houve quaisquer intercorrências.

DISCUSSÃO

O padrão ouro dos materiais de enxertia é a utilização de osso autógeno, devido a suas propriedades altamente osteogênicas, osteocondutoras e osteoindutivas ${ }^{7}$. Porém, o fato de necessitar de um segundo local cirúrgico e ter disponibilidade limitada, resulta em maior desconforto para o paciente e morbidade da área doadora ${ }^{3,10}$.

A hidroxiapatita de cálcio, material aloplástico, atua como osteocondutor facilitando a neoformação óssea ${ }^{11}$. Enquanto o plasma rico em plaquetas e fibrinas, quando associado a outros biomateriais, facilita a incorporação do enxerto ao osso da maxila, acelerando assim a cicatrização ${ }^{11}$. Apesar dos avanços tecnológicos na bioengenharia tecidual, o osso autógeno é ainda considerado o composto de escolha como enxerto sinusal, principalmente associado a outros biomateriais $^{7,11}$.

Todos os biomateriais utilizados no aumento do seio maxilar mostram boa biocompatibilidade e propriedades osteocondutoras podendo ser utilizados com sucesso $^{12}$. No entanto o aloenxerto de osso mineralizado liofilizado aparenta ter o melhor resultado histomorfométrico em termos de osso neoformado e material de enxerto residual 12.

Um estudo relatou que a elevação do seio maxilar com substitutos ósseos sintéticos apresenta altas taxas de sobrevivência de supra estruturas e implantes sem diferenças significativas em comparação com enxerto ósseo autógeno ou xenoenxerto10. Em contrapartida, os substitutos ósseos como o enxerto xenógeno, são os mais escolhidos pelos cirurgiões-dentistas, pois evitam a utilização de um segundo local cirúrgico, diminuindo a morbidade da área doadora e sem limitação em relação à quantidade de material disponível ${ }^{7,2}$.

CONCLUSÃO

O caso apresentado demonstra a viabilidade do levantamento de seio maxilar utilizando enxerto ósseo bovino como biomaterial e a instalação imediata de um implante de conexão cônica na região de enxertia. Alguns fatores são essenciais para 0 sucesso destes procedimentos, como a quantidade de remanescente ósseo, a estabilidade primária do implante no momento da instalação, a manutenção da integridade da membrana sinusal, a saúde do seio maxilar prévio e o tipo de conexão do implante.

\section{REFERÊNCIAS}

1. Lajnert V, Kovacevic Pavicic D, Pavlic A, Pokrajac-Bulian A, Spalj S. Smile Aesthetics Satisfaction Scale: development and validation of a new brief five-item measure of satisfaction with smile aesthetics in adults and the elderly. Int Dent J. 2018;68(3):162-70. 
2. da Silva HF, Goulart DR, Sverzut AT, Olate S, de Moraes M. Comparison of two anorganic bovine bone in maxillary sinus lift: a split-mouth study with clinical, radiographical, and histomorphometrical analysis. Int J Implant Dent. 2020;6(1):17.

3. Galafassi DB, Gomes-Ferreira PHS, Okamoto R, Ferreira S, Momesso GAC, Faverani LP. Instalação de implante imediato à levantamento de seio maxilar com utilização de enxerto autógeno. Arch Health Invest. 2015;4(Spec Iss2):170.

4. Santos KR, Cardoso A, Machado NA, Pinheiro AR, Merly F. Sinus Lift Procedure With Simultaneous Implant Instalation in Patients With Reduced Subantral Space. Int J Sci Dentistry. 2016; 22(46)

5. Trajano B A, Pimentel ACQ, Toda C, Vieira, JMR, Martins LDM. Implantes curtos em região posterior de maxila e mandíbula. BJIHS. 2020;2(3):84-94.

6. Rolim GF, Brito LGC, Silva AA, Saturnino MS, Goldfarb ECA, Sousa TP et al. Levantamento de seio maxilar traumático com o uso de matriz óssea bovina. Braz J Develop. 2020;6(7):53697-713.

7. Albuquerque AFM, Cardoso IML, Silva JSP, Germano AR, Dantas WRM, Gondim ALMF. Levantamento de Seio maxilar utilizando osso liofilizado associado a instalação imediata de implante do tipo cone morse: relato de caso. RFO UPF. 2014;19(1):129-34.

8. Lim EL, Ngeow WC, Lim D. The implications of different lateral wall thicknesses on surgical access to the maxillary sinus. Braz Oral Res. 2017;31:97.

9. Starch-Jensen T, Arne M, Becktor JP, Jensen SS. Maxillary sinus floor augmentation with synthetic bone substitutes compared with other grafting materials. Implant Dent. 2018;27(3):363-74.

10. Santos A, Amorim R, Dietirch L, Costa M, Gontijo J, Silva G. Principais biomateriais utilizados na técnica de levantamento de seio maxilar. ROC. 2020;4(1):42-55

11. La Monaca G, lezzi G, Cristalli MP, Pranno N, Sfasciotti GL, Vozza I. Comparative Histological and Histomorphometric Results of Six Biomaterials Used in Two-Stage Maxillary Sinus Augmentation Model after 6-Month Healing. Biomed Res Int. 2018;2018:9430989.

\section{CONFLITO DE INTERESSES}

Os autores declaram não haver conflitos de interesse

AUTOR PARA CORRESPONDÊNCIA

\section{Camila Melo Pereira}

Faculdade Cathedral, 69307-053 Boa Vista - RR, Brasil

E-mail: camiilaamelo17@gmail.com 